\title{
Anemia em crianças menores de dois anos atendidas nas unidades básicas de saúde no Estado de São Paulo, Brasil
}

\author{
Anaemia in children under two years in basic health care units in the state of \\ S. Paulo, Brazil
}

Marco A. A. Torres*, Kazue Sato*, Suzana de Souza Queiroz**

\begin{abstract}
TORRES, M. A. A. et al. Anemia em crianças menores de dois anos atendidas nas unidades básicas de saúde no Estado de São Paulo, Brasil. Rev. Saúde Pública, 28: 290-4, 1994. Foi realizado estudo com o objetivo de verificar a prevalência da anemia em crianças atendidas nas unidades básicas de saúde do Estado de São Paulo, Brasil. Foram estudadas 2.992 crianças de 6 a 23 meses de idade, atendidas dentro da demanda espontânea, em 160 unidades de saúde de 63 municípios das 5 Coordenações das Regiões de Saúde do Estado (CRS). O sangue foi coletado por punção venosa, e a hemoglobina dosada pelo método da cianometa-hemoglobina. Utilizou-se o critério da Organização Mundial de Saúde para caracterizar a anemia ( $\mathrm{Hb}<11,0 \mathrm{~g} / \mathrm{dl}$.). Detectou-se que $59,1 \%$ das crianças eram anêmicas, sendo que a prevalência variou entre $47,8 \%$ e $68,7 \%$ nas 5 CRS. A CRS-1, que compreende a Região Metropolitana da Grande São Paulo, apresentou prevalência de anemia significantemente inferior à observada nas 4 CRSs que se situam no interior do Estado. Encontrou-se níveis de hemoglobina inferiores a $9,5 \mathrm{~g} / \mathrm{dl}$ em $25,1 \%$ das crianças. A anemia atingiu mais as crianças do sexo masculino, as que nasceram com peso inferior a $3.000 \mathrm{~g}$, as que foram amamentadas por um período inferior a 2 meses e as que apresentavam algum grau de desnutrição energético-protéica, segundo o critério de Gomez.
\end{abstract}

Descritores: Anemia hipocrômica, epidemiologia. Hemoglobinas, análise.

\section{Introdução}

Até há bem pouco tempo, a carência nutricional de maior prevalência, em todo o Brasil, era a Desnutrição Energético-Protéica (DEP) ${ }^{1,15}$. Entretanto, esta realidade vem se alterando nos últimos anos. Em 1974, 22\% das crianças menores de 5 anos, na cidade de São Paulo, foram classificadas como anêmicas ${ }^{14}$, ou seja, apresentavam níveis de

* Grupo de Atenção à Nutrição do Centro de Apoio ao Desenvolvimento da Atenção Integral à Saúde e Núcleo de Nutrição do Centro de Referência de Saúde da MuIher, Nutrição, Alimentação e Desenvolvimento Infantil da Secretaria de Estado da Saúde de São Paulo

** Departamento de Pediatria da Faculdade de Medicina de Botucatu. Universidade Estadual Paulista/UNESP e Grupo de Atenção à Nutrição do Centro de Apoio ao Desenvolvimento da Atenção Integral à Saúde e Núcleo de Nutrição do Centro de Referência de Saúde da Mulher, Nutrição, Alimentação e Desenvovimento Infantil da Secretaria de Estado da Saúde de São Paulo, São Paulo, SP - Brasil

Separatas/Reprints: M. A. A. Torres. - R. Dr. Augusto de Miranda, 1297 - 05026-001 - São Paulo, SP . Brasil

Edição subvencionada pela FABESP. Processo 94/0500-0. hemoglobina inferiores a $11,0 \mathrm{~g} / \mathrm{dl}$. Dez anos após, este percentual subiu para 35,5\%10. Em 1989, o Grupo de Atenção à Nutrição (GAN) da Secretaria de Estado da Saúde de São Paulo, estudando crianças de 9 a 23 meses de idade, atendidas em duas unidades de saúde no Município de São Paulo, encontrou $50 \%$ de anemia, sendo que, aproximadamente, $95 \%$ delas eram devidas à carência de ferro ${ }^{16}$.

Em Recife, capital do Estado de Pernambuco, $82,8 \%$ das crianças entre 6 e 23 meses de idade, atendidas em uma unidade de saúde, apresentaram anemia ${ }^{13}$. No Estado do Pará, a prevalência situase em torno de $70 \%{ }^{9}$.

Pode-se afirmar que, atualmente, a anemia é o problema nutricional de maior magnitude, não só no Brasil, como em todo o mundo, afetando mais de 700 milhões de pessoas ${ }^{3}$, principalmente crianças, gestantes e lactantes e a carência de ferro é o seu principal agente etiológico ${ }^{1,2,3,11}$.

O Estado de São Paulo, segundo dados do $\operatorname{IBGE}^{4}$ (1991), possui 628 municípios e uma população de 34.207.957 habitantes, correspondente a $23,4 \%$ da população brasileira. Metade desta população reside na segunda maior área metropolitana do mundo, a Grande São Paulo, que ocupa uma área correspondente a 3,2\% do total do Estado e é 
composta por 38 municípios, incluindo a cidade de São Paulo 5 .

Frente ao aumento da prevalência de anemia verificada na cidade de São Paulo e à ausência de informações sobre a situação nas demais regiões do Estado, considerou-se fundamental a realização do presente estudo, idealizado com o objetivo de verificar a prevalência de anemia em crianças atendidas nas unidades básicas de saúde do Estado de São Paulo; determinar as diferenças existentes em cada uma das Coordenações de Regiões de Saúde (CRS); e relacionar a presença de anemia com idade, sexo, peso ao nascer; estado nutricional e tempo de aleitamento materno das crianças estudadas.

\section{Material e Método}

A Secretaria de Estado da Saúde de São Paulo está dividida em 5 macro-regiões administrativas: as Coordenações das Regiões de Saúde (CRS). Cada CRS é composta por, aproximadamente, 15 Escritórios Regionais de Saúde (ERSAS) que, por sua vez, tem sob sua administração 10 a 15 municípios, conforme o mapa da Figura 1.
Foram estudadas 2.992 crianças, aproximadamente 600 por CRS, com idades compreendidas entre 6 e 23 meses, em 160 unidades de saúde de 63 municípios nas 5 CRS (Tabela 1). O desenho amostral permitiu inferir conclusões significativas em nível de cada CRS, com uma margem de erro de $5 \%$. O número de crianças estudadas em cada município foi determinado pelo peso que sua população representava na somatória da população total dos municípios selecionados, em cada CRS.

A amostra foi escolhida aleatoriamente entre a clientela que procurava espontaneamente a unidade de saúde, para atendimento médico. Após autorização dos pais ou responsáveis, era preenchido

Tabela 1 - Distribuição da amostra estudada em cada Coordenação Regional de Saúde

\begin{tabular}{ccc}
\hline CRS & Amostra & $\%$ \\
\hline 1 & 536 & 17,9 \\
2 & 591 & 19,8 \\
3 & 629 & 21,0 \\
4 & 603 & 20,1 \\
5 & 633 & 21,2 \\
\hline Total & 2.992 & 100,0 \\
\hline
\end{tabular}

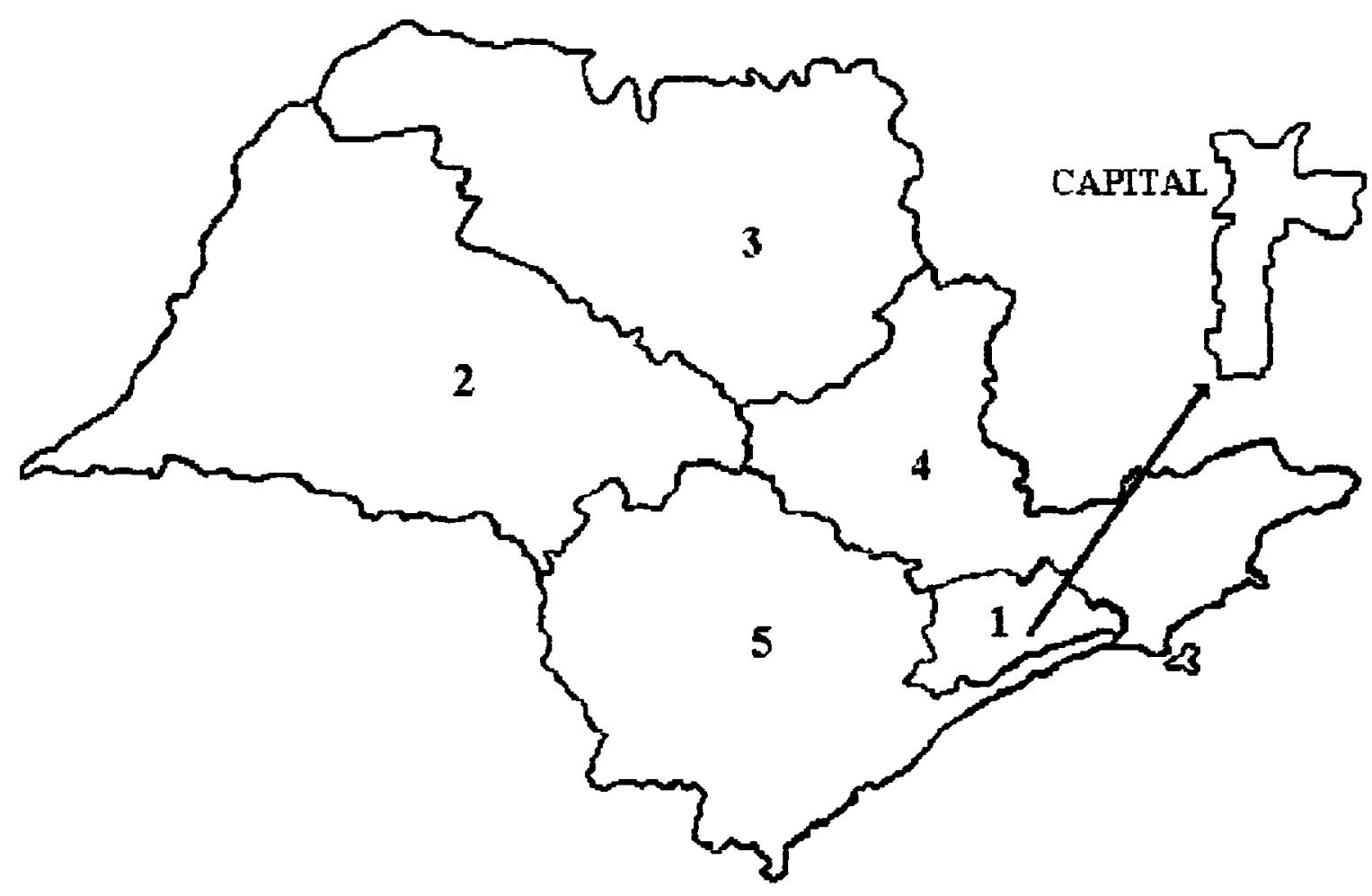

Figura 1 - Mapas das macro-regiōes de saúde do Estado de São Paulo 
um questionário e a seguir realizada a coleta do sangue para dosagem da hemoglobina.

O questionário continha informações sobre as condições de nascimento, amamentação, sexo, idade, bem como peso e estatura da criança no dia da entrevista.

O sangue era coletado por punção venosa e a dosagem da hemoglobina era feita pelo método da cianometa-hemoglobina ${ }^{7}$, com leitura em duplicata, feita em espectrofotômetro. Com a finalidade de eliminar erros decorrentes de leituras realizadas em diversos aparelhos, utilizou-se, para o cálculo da hemoglobina, um fator único preconizado pela "International Commitee for Standardization in Hematology"8.

Para a caracterização de anemia foi adotado o critério da Organização Mundial de Saúde (OMS) que considera anêmica a criança, com idade compreendida entre 6 meses e 6 anos, que apresente dosagem de hemoglobina inferior a $11,0 \mathrm{~g} / \mathrm{dl}^{11}$. O estado nutricional foi avaliado pelo critério de Go$\mathrm{mez}^{6}$, utilizando-se como padrão de referência as recomendações da $\mathrm{OMS}^{12}$.

Para a análise estatística foi utilizado o teste não paramétrico do $\mathrm{X}^{2}$, para Tabelas $2 \times 2$ e $2 \times \mathrm{n}$.

\section{Resultados}

A Figura 2 mostra que $59,1 \%$ das crianças com idades entre 6 e 23 meses, atendidas nas unidades básicas de saúde do Estado de São Paulo, apresentam anemia. As prevalências variam de $47,8 \%$ na CRS-1 (Grande São Paulo) até 68,7\% na CRS-4 (leste do Estado). Na CRS-3 (norte do Estado) constata-se a segunda maior prevalência, $63,3 \%$, seguida da CRS-2 (noroeste do Estado) com $58,1 \%$ e da CRS-5 (região sudoeste), com 56,4\%.

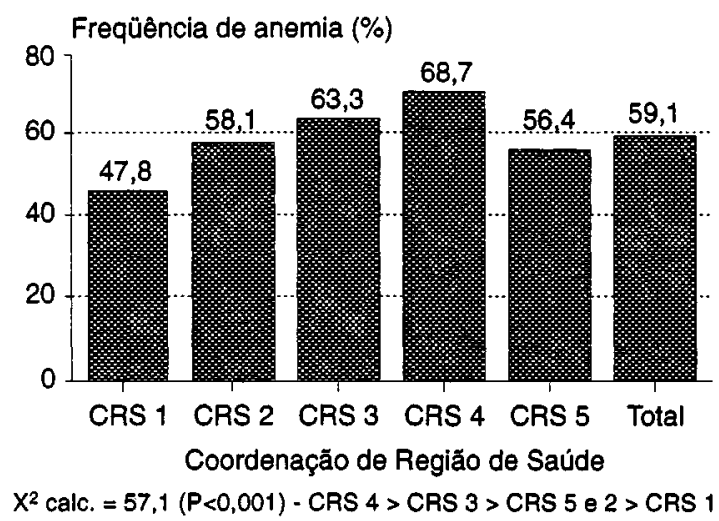

Figura 2 - Prevalência de anemia, em crianças de 6 a 23 meses atendidas nas unidades básicas de saúde, segundo a Coordenação Regional de Saúde (CRS), do Estado de São Paulo, 1993.

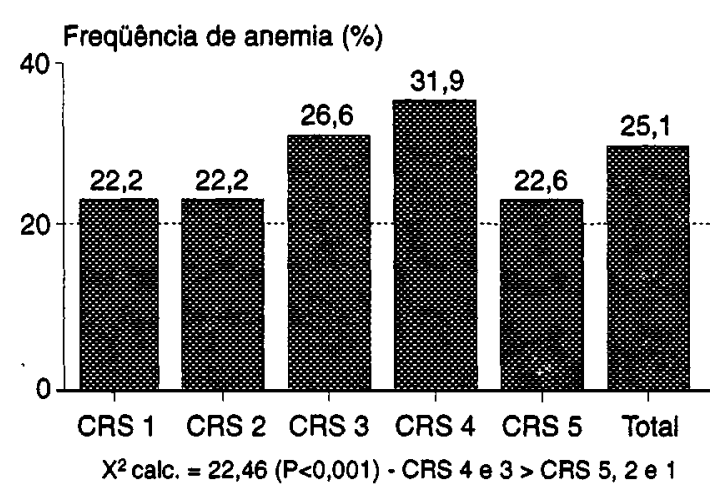

Figura 3 - Percentagem de crianças menores de 2 anos, com anemia grave, por Coordenação de Região de Saúde (CRS) no Estado de São Paulo.

A região metropolitana (CRS-1) é a que apresenta os menores índices, quando comparada com as CRS que representam o interior do Estado. O teste do $\mathrm{X}^{2}$ mostra que esta diferença é significante $(\mathrm{P}<0,001)$.

Tomando-se o ponto de corte da hemoglobina em $9,5 \mathrm{~g} / \mathrm{dl}$ verifica-se que $25,1 \%$ das anemias se situam abaixo deste nível (Figura 3). As CRS-1, 2 e 5 apresentam prevalências praticamente iguais $(22,2 \%$

Tabela 2 - Relação entre a presença de anemia e algumas variáveis estudadas em crianças menores de 2 anos atendidas

\begin{tabular}{|c|c|c|}
\hline - Variável & $\%$ de Anemia & Teste do X2 \\
\hline $\begin{array}{l}\text { Sexo* } \\
\text { Masculino } \\
\text { Feminino }\end{array}$ & $\begin{array}{l}63,1(A) \\
55,2(B)\end{array}$ & $\begin{array}{l}X^{2}=18,82 \\
A>B(P<0,001)\end{array}$ \\
\hline $\begin{array}{l}\text { Idade* } \\
6 \text { a } 8 \text { meses } \\
9 \text { a } 11 \text { meses } \\
12 \text { a } 17 \text { meses } \\
18 \text { a } 23 \text { meses }\end{array}$ & $\begin{array}{l}55,5(A) \\
62,8(B) \\
62,8(C) \\
55,8(D)\end{array}$ & $\begin{array}{l}X^{2}=16,66 \\
A \in D<B \in C \\
(P<0,001)\end{array}$ \\
\hline $\begin{array}{l}\text { Peso ao Nascer** } \\
<2.500 \mathrm{~g} . \\
2.500 \geq 2.999 \mathrm{~g} . \\
\geq 3.000 \mathrm{~g} .\end{array}$ & $\begin{array}{l}67,9(\mathrm{~A}) \\
62,1(\mathrm{~B}) \\
55,0(\mathrm{C})\end{array}$ & $\begin{array}{l}X^{2}=32,31 \\
A \in B>C \\
(P<0,001)\end{array}$ \\
\hline $\begin{array}{l}\text { Aleitamento Matern } \\
\text { Zero } \\
1 \text { e } 2 \text { meses } \\
3 \text { a } 5 \text { meses } \\
6 c+\text { meses }\end{array}$ & $\begin{array}{l}62,7(\mathrm{~A}) \\
60,6(\mathrm{~B}) \\
57,3(\mathrm{C}) \\
55,5(\mathrm{D})\end{array}$ & $\begin{array}{l}X^{2}=8,56 \\
A \in B>C \ominus D \\
(P<0,05)\end{array}$ \\
\hline $\begin{array}{l}\text { Estado Nutricional ( } \\
\text { Normal } \\
\text { D-I } \\
\text { D-II + D-III }\end{array}$ & $\begin{array}{l}\text { Gomez)* } \\
\qquad \begin{array}{l}\text { 56,3 (A) } \\
61,9(\mathrm{~B}) \\
73,6(\mathrm{C})\end{array}\end{array}$ & $\begin{array}{l}X^{2}=21,98 \\
A<B<C \\
(P<0,001)\end{array}$ \\
\hline
\end{tabular}


e 22,6\%), existindo diferenças significantes $(\mathrm{P}<0,001)$ quando comparadas com as observadas nas CRS 3 e $4, \operatorname{com} 26,6 \%$ e $31,9 \%$, respectivamente.

A Tabela 2 apresenta a relação encontrada entre a presença de anemia e algumas variáveis biológicas consideradas. Para a idade, sexo e condição nutricional os resultados referem-se ao total da amostra estudada (2.992 crianças); em relação aos itens peso ao nascer e tempo de aleitamento materno considerou-se apenas a população de menores de um ano (1.412 crianças), uma vez que o reflexo destas duas variáveis sobre as condições de saúde da criança são muito maiores durante o primeiro ano de vida.

Verifica-se que a anemia atinge mais as crianças do sexo masculino $(63,1 \%)$ que as do feminino $(55,2 \%)$, com o teste do $X^{2}$ mostrando uma associação estatisticamente significante $(\mathrm{P}<0,001)$.

Nas faixas etárias de 6 a 8 e de 18 a 23 meses foram detectadas, respectivamente, $55,5 \%$ e $55,8 \%$ de crianças anêmicas, percentual significantemente inferior $(\mathrm{P}<0,001)$ ao encontrado em crianças de 9 a 11 e de 12 a 17 meses, $62,8 \%$.

As crianças menores de um ano que nasceram com peso inferior a $2.500 \mathrm{~g}$ apresentam mais anemia $(67,9 \%)$ que as que nasceram com peso entre $2.500 \mathrm{~g} \mathrm{e} 3.000 \mathrm{~g}(62,1 \%)$. Este percentual decresce a $55,0 \%$ se a mesma nasceu com peso superior a $3.000 \mathrm{~g}(\mathrm{P}<0,001)$.

Também na população de menores de um ano detectou-se que existe associação estatisticamente significante $(P<0,05)$ entre a presença de anemia e o tempo de aleitamento materno. A prevalência é de $62,7 \%$ se a criança não foi amamentada ao seio, $60,6 \%$ se foi amamentada até 2 meses, $57,3 \%$ se mamou entre 3 e 6 meses e $55,5 \%$ se este período foi superior a 6 meses.

A condição nutricional, pelo critério de Gomez, mostra que $56,3 \%$ das crianças normais apresentam anemia. Este percentual sobe para $61,9 \%$, se ela for desnutrida de grau I, e para $73,6 \%$ nas desnutridas moderadas ou graves (D-II e D-III). O teste do $\mathrm{X}^{2}$ fortalece a relação existente entre a presença de anemia e a piora da condição nutricional da criança $(\mathrm{P}<0,001)$.

\section{Discussão}

Os resultados apresentados reforçam o fato da anemia nutricional por carência de ferro ser a patologia de maior prevalência na população com menos de 2 anos em todo o Estado de São Paulo, superando a DEP, que atinge $35 \%$ das crianças estudadas. Embora este não seja um estudo populacional, acredita-se que os resultados não se distanciam muito da situação real, uma vez que é alta a cobertura populacional das uni- dades básicas de saúde, principalmente no interior do Estado.

As diferenças observadas nas regiões do Estado mostram que, ao contrário do que se poderia supor, a região metropolitana da Grande São Paulo (CRS-1) é a que mostra as menores prevalências. No interior, as áreas economicamente mais desenvolvidas (CRS-4 e CRS-3) registraram os maiores índices. A CRS-5, a mais pobre do Estado, apresenta os menores índices do interior.

A situação apresentada é de difícil explicação com os dados dísponíveis no presente trabalho. Acredita-se serem necessários estudos complementares sobre a epidemiologia da anemia no Estado de São Paulo. Podemos, entretanto, tecer algumas considerações. É um fato bastante conhecido que a carência de ferro acomete, de maneira muito importante, indivíduos que se encontram em fases de crescimento acelerado, com conseqüente aumento das necessidades de ferro. Neste raciocínio, pode-se supor, conforme já colocado por diversos autores ${ }^{2,17}$, que crianças acometidas de Desnutrição Energético-Protéica, sem intercorrências que levem a grandes espoliações, têm menor necessidade deste oligoelemento, em função da desaceleração de seu crescimento. No presente levantamento, constatou-se que as CRS 1 e 5 apresentaram as maiores prevalências de DEP, justamente onde foram registrados os menores índices de anemia.

A maior ocorrência de anemia no sexo masculino talvez possa ser explicada pela maior velocidade de crescimento apresentada pelos meninos, nesta faixa etária, acarretando maior necessidade de ferro pelo organismo, não suprida pela dieta.

Não foi encontrada diferença entre a presença de anemia nas faixas etárias de menores e maiores de um ano de idade. Estes dados concordam com os encontrados por Romani e col. ${ }^{13}$, em Recife. Existem, entretanto, registros na literatura que apontam maiores freqüências (em torno de 50\%) na faixa etária dos 9 a 23 meses, no Município de São $\mathrm{Paulo}^{16}$, o que pode ser indicativo de que, além de estar se instalando mais precocemente, a ocorrência de anemia continua aumentando, principalmente em menores de um ano.

Os dados observados no presente estudo reforçam a literatura que coloca o recém-nascido de baixo peso, por desnutrição intra-uterina ou prematuridade, como um dos fatores predisponentes mais importantes na gênese da carência de ferro, pela redução das reservas acumuladas durante a gestação ${ }^{2}$.

A anemia pode ser assinalada como uma carência nutricional que pode ocorrer independentemente da DEP. Existem, entretanto, série de fatores que evidenciam a forte relação encontrada entre estas duas patologias (baixa ingestão de nu- 
trientes, infecções repetidas, redução da absorção de ferro por comprometimento da mucosa intestinal, entre outras).

Os dados apresentados no presente estudo revelam uma situação preocupante, que reclama medidas urgentes e eficazes para o controle da situação, sob pena de prejuízos irreversíveis para as gerações futuras.

\section{Agradecimentos}

Aos técnicos e funcionários da Secretária de Estado da Saúde de São Paulo que, nas unidades básicas de saúde, Prefetituras Municipais, Escritórios Regionais de Saúde e Coordenação de Regiões de Saúde possibilitaram e participaram da realização do estudo; aos Profs. Drs. Neil Ferreira Novo e Yara Juliano, da Escola Paulista de Medicina, pelo tratamento estatístico.

TORRES, M. A. A. et al. [ Anaemia in children under two years in basic health care units in the State of $S$. Paulo, Brazil]. Rev. Saúde Puiblica, 28: 290-4, 1994. The present study sought to determine the prevalence of anemia in 2,992 children, aged between 6 and 23 months, who voluntarilly attended 160 Basis Health Care Units, located in 63 cities of the 5 Regional Health Coordinating areas of the State of S. Paulo, Brazil. Blood samples were collected by venous puncture and hemoglobin was measured by the cianometahemoglobin method. The WHO criteria for the diagnosis of anemia $(\mathrm{Hb}<11.0 \mathrm{~g} / \mathrm{dl})$ were used: $59.1 \%$ of the children were shown to be anemic, with prevalence varying from $47.8 \%$ to $68.7 \%$ in the 5 RHCs. RHC 1, which comprises the Greater S. Paulo Region, presented a prevalence of anemia significantly lower than the other $4 \mathrm{RHCs}$, wich cover the rest of the State. Hemoglobin levels $9.5 \mathrm{~g} / \mathrm{dl}$ were found in $25.1 \%$ of the children. Anemia was more frequent in male children in male children, in those born, with a weight of less than $3,000 \mathrm{gr}$, in those who were breastfed for less than 2 months and in those that who presented some degree of energy deficient proteic malnutrition, according to Gomez's criteria. This is the first of a series of 4 articles whose purpose is the determining the prevalence of anemia in the State of S. Paulo and of testing the intervention alternatives with a view to curtailing the incidence of this pathology which today is the most prevalent nutritional disturbance in the world.

Keywords: Anemia, hypochromic, epidemiology. Hemoglobins, analysis.

\section{Referências Bibliográficas}

1. BATISTA FILHO, M. \& BARBOSA, N.P. Pró-memória. Alimentação e Nutrição no Brasil: 1974-1984. Brasília Ed. e Gráfica Canadá, 1985.

2. BRANDALISE, S.R. \& MATSUDA, E. Anemias carenciais. In: NÓBREGA, F.J. Desnutrição Intra-uterina e pósnatal. São Paulo, Panamed Ed., 1986. p. 427-37.

3. DeMAYER, E.M. Preventing and controling iron deficiency anaemia through primary care. Ginebra, OMS, 1989.

4. FUNDAÇĀO IBGE. Anuário estatístico do Brasil: resenha histórica. Rio de Janeiro, 1991.

5. FUNDAÇÃO SEADE. Anuário estatístico do Estado de São Paulo - 1991. São Paulo, 1991.

6. GOMEZ, F. Desnutrición. Bol. Méd. Hosp. Infant. Méx., 3: 543-51, 1946.

7. HAILINE, A. Standard methods of clinical chemistry. 1958.v. 2.

8. INTERNATIONAL COMMITTEE FOR STANDARDIZATION IN HEMATOLOGY. Standard techiniques for measurement of red cell and plasma volume. Br.J. Haematol, 25: 801, 1973.

9. JOÃO, W.S.J. Prevalência de anemia na população atendida nos postos de saúde da Secretaria de Saúde do Estado do Pará e em escolares participantes do Programa Nacional de Alimentação Escolar - relatório anual. Belém, 1983. [Convênio INAN/UFPA].

10. MONTEIRO, C.A. Saúde e nutrição das crianças de São Paulo: diagnóstico, contraste sociais e tendências. São Paulo, HUCITEC / EDUSP, 1988.

11. ORGANIZACION MUNDIAL DE LA SALUD. Lucha contra la anemia nutricional, especialmente contra la carencia de hierro. Ginebra, 1975. (OMS Serie de Informes Tecnicos, 580).

12. ORGANIZACION MUNDIAL DE LA SALUD. Medición del efecto nutricional de programas de suple. mentación alimentaria a grupos vuluerables. Ginebra, 1980.

13. ROMANI, S.A.M. et al. Anemias em pré-escolares diagnóstico, tratamento avaliação - Recife-Pe, Brasil. Arch. Latinoam. Nutr, 67 : 159-67, 1991.

14. SIGULEM,D.M. et al. Anemia ferropriva em crianças no Município de São Paulo. Rev. Suíde Pública., 12: 168 . 78, 1978.

15. TORRES, M.A.A. Estado nutricional e aspectos sócioeconômicos de familias rurais do Trópico Semi-Árido (nordeste do Brasil). Recife, 1982. [Dissertação de Mestrado - Universidade Federal de Pernambuco].

16. TORRES, M.A.A., SATO, K., SOUZA QUEIROZ, S. A, Terapêtutica com doses profiláticas de sulfato ferroso, como medida de intervenţăo no combate à carência de ferro en crianças atendidas em unidades básicas de saúde. São Paulo, Secretaria de Estado da Saúde, 1989. [dados inéditos].

17. VIART, P. Blood Volume $(51 \mathrm{Cr})$ in severe protein-caloric malnutrition. Am. J. Clin. Nutr., 29: 25, 1976.

Recebido para publicasão em 17.2.1994 Aprovado para publicação em 6.6.1994 